\title{
An Overview of the Neurological Base of Bipolar Disorder
}

\author{
Maria Santiago Valentín*
}

Somerset County Vo-Tech Schools, Child Study Team, 14 Vogt Drive Bridgewater, NJ 07066, USA

*Corresponding author: Maria Santiago Valentín, Learning Disabilities Consultant, Somerset County Vo-Tech Schools, Child Study Team, 14 Vogt Drive Bridgewater, NJ 07066, USA, Phone: + 732-9126376; E-mail: Msant860@aol.com

Received date: Oct 07, 2017; Accepted date: Oct 23, 2017; Published date: Oct 27, 2017

Citation: Valentín MS (2017) An Overview of the Neurological Base of Bipolar Disorder. J Child Dev Disord. Vol 3. No 4: 20.

Copyright: (C) 2017 Valentín MS. This is an open-access article distributed under the terms of the Creative Commons Attribution License, which permits unrestricted use, distribution, and reproduction in any medium, provided the original author and source are credited.

\section{Introduction}

\section{Etiology of the Disorder from the Neurological Perspective}

Bipolar Disorder has been affecting us for centuries and it has carried different names such as: melancholia, madness, psychopathic temperament, maniac-depression, among others. This paper attempts to give an overview of the neurological basis of this condition, how the medical approaches have evolved through centuries, provide some insight on the variety of treatments that sometimes are just part of a guessing game to find a solution and how we as educators can impact the learning experience of a student with Bipolar Disease by looking at accommodations that must take place in the classroom. The first signs in history of this disease appeared in 300-500 AD. The writings of a Greek named Aretaeus of Cappodociam are the first documents found describing the mania and depressive episodes he saw in his patients. "In the earliest days of documentation, these people were viewed as 'crazy,' possessed by the devil or demons, Dr. Gardenswartz says. Their treatment or punishment, she explains, included restraint or chaining; their blood was let out; they were given different potions, or electric eels were applied to the skull-much in the way witches have been treated in various cultures. In fact, witchcraft was often used to try and 'cure' them," Gardenswartz says" (Stevens 2012). Some cultures today unfortunately due to lack of access to accurate information or misinformation still perceive and approach mental illness with superstition. It was not until the $18^{\text {th }}$ century that a more healthy approach was given to patients of melancholia. The first name that appears is Richard Burton a British scientist. His book Anatomy of the Melancholia written in 1650 gives a detailed description of the symptoms and signs. In the $19^{\text {th }} \mathrm{c}$ in the observations made by the French Jules Falret in 1854 appears the term "folie circulaire" that refers to a dual form of insanity referring to the cyclic episodes of the bipolar disorder. In 1875 his findings were renamed as Manic-Depressive Psychosis. "Another lesserknown fact attributed to Falret is that he found the disease seemed to be found in certain families thus recognizing very early that there was a genetic link" (Stevens 2012). French François Baillarger contribution was the separation of bipolar disorder from other mental illnesses. Then in the $20^{\text {th }}$ century the doctors followed the approach of the German Psychiatrist Emil Krapelin who in 1913 studied the depression episode more than the mania episode. The lack of medication and appropriate treatment caused that many patients spent most of their time in asylums and hospitals for months or years with the indication of not discharging them too early for the risk of suicide. Up to this moment the illness was linked to a genetic factor and family history until the German psychoanalyst Sigmund Freud pushed biology to the side and implicated that the diseased was potentially caused by a childhood traumatic experience or a psych development conflict that was unresolved. He carefully studied the patients' biographies and life events to find conflicts that would be considered triggers of the BD. He saw BD as an illness of the mind and not outcome of the composition of the brain. Here we have the binary opposition of (genetics) nature vs. nurture (environment), in the early 1930s. The psychotherapy developed by Freud was embraced but it did little for the patients of schizophrenia. In Australia John Cade believed that the illness was biological and not psychological and he studied the urine of patients of BD looking for a chemical. In his research he combined uric acid with lithium and injected guinea pigs that became lethargic and unresponsive for two hours. He injected lithium to ten patients who improved. At this point we see a progress in the approach much closer to our time. Other medications used at that time were morphine, quinine, and cod-liver oil but they had no impact on serious illnesses until lithium came into the picture. One of Cade's patient stopped using lithium and the old symptoms reappeared. He was hospitalized, medicated again on Lithium and released with the patient's understanding that daily medication was mandatory. However the use of lithium in the United States took decades since psychotherapy overtook the psychiatric approach of the illness, it was Ronald Fieve who implemented the use of lithium in his practice 1970s. Lithium not only treats the mania episodes, it also prevents their recurrence in BD patients. The fact that lithium made the mania episodes disappear validates the hypothesis that BD is the result of some biochemical relation in the brain of the patient. A 1952 article from The Journal of Nervous and Mental Disorder brings back biology and reaffirms the hypothesis that bipolar disorder runs in families with a history of mental illness. During the 1950's and 1960's all the research 
and study of the genetics and symptoms of BD aimed to distinguish BD from other mental illnesses. Another milestone happened in 1960, when BD was clearly separated from Unipolar D, and eventually the acceptance of 2 types of BD. In the 1970's laws were enacted to help those who suffered from BD, and in 1979 the National Association of Mental Health (NAMI) was founded. One big change is the elimination of the term manic depressive disorder and having it replaced by the term BD. The trend towards genetics is stronger than the hypothesis that environment is the cause. It may trigger the predisposition to the illness, but it can not be considered the leading factor. Educational terms Behaviors and Symptoms [1-10].

According to the most recent version of the Diagnostic and Statistical Manual Description- DSM IV the Bipolar Disorder (DSM-IV-TR \#296.0-296.89) is described clinically as an illness that presents two episodes: a manic episode and a depressive episode. The onset of the first episode has happened to some patients when they are 10 years old. The manifestation of the first episode decreases after the twenties years of age. The manic episode has 3 stages: three stages of mania: hypomania, acute mania, or delirious mania. The mania episodes can last from days to weeks and the signs that indicate the beginning of this state are irritability, pressure of speech, decrease need of sleep and sudden euphoria. The patient experiences the three stages and at the end they look back and feel guilty of the decisions and behavior displayed when they were in this stage that can last from hours to months. In the Hypomania state the patient's confidence and self-esteem is very high mixed with feelings of grandiosity. According to (DSM-IV-TR \#296.0-296.89) "Patients become demanding, inconsiderate, and intemperate. They are constantly dissatisfied and intolerant of others, and brook no opposition" (Boland 2010). It is a very risky stage since the patient lacks of sound judgment. Shopping sprees, hyper-sexuality and conflicts with the law are situations they will regret later. Acute mania is marked occasionally with delusions, and isolated hallucinations. They feel their lack of success is someone else's fault. The grandiose behavior begins to fragment and the patient turns hostile, aggressive. The patient can torn clothes apart, destroy furniture, scream. They may openly insinuate to strangers; strength may be increased, and sensitivity to pain may be lost. The delirious mania is marked with more confusion and hallucinations. The flow of ideas and speech becomes more fragmented. Impulsivity increases and the desire to commit suicide also. The patient loses her/his selfcontrol. In Bipolar 1 the patient mania stages escalate and in BPD 2 the patient only experienced the hypomania stage of the maniac episode. The depressive episode is marked by a complete lack of energy, and interest in life. The patient has difficulty remembering what they read and have trouble concentrating. He becomes pessimistic and recurrent thoughts of suicide appear. The manic-mixed episodes are not to be confused with the transition from mania to depression. These episodes begin with symptoms of mania and depression combined, continue with them, and end with them, and are neither immediately preceded nor immediately followed by a mania or depressive episode. Alcoholism and cocaine addiction are two disorders related to $B D$ that can interfere in the correct diagnosis of this illness. We see a trend that the acute the mania, the severe the psychosis is in the patient. The etiology from the neurological perspective has the following findings: genes play a role in bipolar disorder, that the prevalence for $B D$ is higher among first degree relatives of the patients, in other words parents and children, gran-parents and grand-children. Autopsy studies have revealed a reduced neuronal number in the locus ceruleus and median raphe nucleus. The locus ceruleus is a small nucleus located in the pons and its neurons are of medium size. The pons is located in the brain stem, above the medulla, below the midbrain, and before the cerebellum. Its job is to regulate the tempo of the brain and release norepinephrine. It is linked to the limbic system through the hypothalamus that regulates our emotions. Fewer neurons in the locus ceruleus will potentially impact the communication among them when it comes to the emotions and it may explain the hallucinations, delusions, over irritability and hyper-sexuality the patient experiences during the mania and the depressive episodes. Median raphe nucleus is responsible for releasing serotonin and having a fewer neuronal number can be correlated to the insufficient production or shortage of this substance in Bipolar Disorder. However, doctors found too simplistic the explanation of the serotonin production and found that medication for depression was blocking the reuptake pump of the neurotransmitters in the synapse. Therefore the doctors concluded at that time that depression is caused by a lower level of neurotransmitter while mania was induced by a too high level of neurotransmitter. Lithium appears to not affect the neurotransmitters at all since it seems to target the inside of the neuron. There are proteins in the neuron called $G$ proteins that are second messengers in the nervous system and that protein inside the cell is what lithium targets. Recent research has found that the amygdala that tells us about danger tends to respond more in patients with BD. Pre-frontal cortex with the amygdala seems to be too active in people with $\mathrm{BD}$ while the lateral pre-frontal cortex, the rational part is less active in people with BD. That leads to the hypothesis that people with $\mathrm{BD}$ see the world in more emotional terms and experience it in such way. Another approach to explain BD is the current mentioning of neuroplasticity, "the neurons responsiveness and ability to react and change to stress" (Mondimore 2006) is disrupted. Research mentions problems with the neurons that make the electrical impulse of the serotonin and norepinephrine either too high or low resulting in extreme emotional moods. Brain imaging in animals has shown that animals injected with lithium, and antidepressants show a growth of neurons in the hippocampus. "For example, we have evidence that several antidepressants foster the growth of connections between brain cells, and we believe this is related to the beneficial effects of these medications Lithium -a naturally occurring element, not really a "drug"-may help in bipolar disorder by protecting damaged brain cells and promoting their ability to communicate with each other" (Machado, Husseini and Zarate 2011). These findings question the accepted hypothesis that after adulthood the brain stops producing new neurons. If the neurons experience plasticity "antidepressants may artificially change the neurotransmitters 
levels in the synapse, which turns on the neurons to start making molecules and other cell components needed to retune existing neurons and grow new ones" (Mondimer 2006) [11-16].

\section{Treatments Indicated for This Disorder and Cutting Edge Approaches}

Bipolar disorder requires lifelong treatment, even during periods when the patient feels better. Treatment is usually treated by a psychiatrist. The patient may have a team that includes a psychologist, or a social worker. The primary treatments for bipolar disorder include medications; individual, group or family psychological counseling (psychotherapy); and/ or education and support groups. "The overall treatment of bipolar disorder is conveniently approached by considering, the treatment of the manic or mixed-manic episode first, then the treatment of the depressive episode, in each instance considering three phases of treatment: acute, continuation, and preventive. As will be seen, of all the medications useful in bipolar disorder, lithium is probably the best choice as it is the only one which has been shown to be effective for all three phases of treatment for both manic and depressive episodes" (Boland 2010). Lithium protects against depression and mania and it decreases the risk of suicide and short-term mortality. Lithium is used for the acute phase, the second mood stabilizer is Divalproex. It is effective in a couple of days, while Lithium takes longer time, usually one or two weeks. Some BD patients need to be locked in a closed unit, and those under acute mania need to be secluded. Continuation treatment consists of avoiding a breakthrough of symptoms while the episode stills on. Lithium is still used in this phase while blood tests are done because when mania is controlled, the blood levels rise as an indicator. Treatment should continue if the patient's life is unstable, and if lithium was prescribed, it is important to continue the dose over a few weeks time, since abrupt discontinuation of lithium predisposes to a recurrence of mania. Preventive treatment continues with mood stabilizers, and with psychotherapy. Olanzapine seems to be effective in the preventive treatment. For the depressive episode is important the patient takes a mood stabilizer at the same time to avoid the risk of an antidepressant triggering the mania episode. Other medication includes: Anticonvulsants. Mood-stabilizing medications include valproic acid (Depakene, Stavzor), divalproex (Depakote) and lamotrigine (Lamictal). The medication asenapine (Saphris) may be helpful in treating mixed episodes. Common side effects include weight gain, dizziness and drowsiness. Rarely, other serious problems, such as skin rashes, blood disorders or liver problems. Antipsychotics. Such as aripiprazole (Abilify), olanzapine (Zyprexa), risperidone (Risperdal) and quetiapine (Seroquel), may help people who don't benefit from anticonvulsants. The only antipsychotic approved by the U.S. Food and Drug Administration (FDA) for treating BPD is quetiapine. Common side effects include weight gain, sleepiness, memory loss, involuntary body/ facial movements. Antidepressants. Sometimes antidepressants can trigger manic episodes, but must be taken along with a mood stabilizer. Symbyax. It works as a depression treatment and a mood stabilizer. Symbyax is approved by the FDA and some side effects can include weight gain, drowsiness and increased appetite. Benzodiazepines are anti-anxiety medications that help with anxiety and improve sleep. Examples are clonazepam (Klonopin), lorazepam (Ativan), diazepam (Valium), chlordiazepoxide (Librium) and alprazolam (Niravam, Xanax). Some side effects can include drowsiness, reduced muscle coordination, and problems with balance and memory.

Psychotherapy is another part of BD treatment. There are several types of therapy and these include: Cognitive behavioral therapy. The focus of this therapy is to identify unhealthy, negative beliefs and behaviors and replace them with healthy, positive ones. Psychoeducation. It is counseling to help the patient learn about and understand BPD. Another approach is Family therapy. It involves the patient seeing a psychologist along with family members to reduce stress within the family as well as solve problems and resolve conflicts. Group therapy provides the platform to communicate with and learn from others with BPD.

Other treatments that do not require medication or psychotherapy are explained in the following paragraphs. Electroconvulsive therapy can be effective for people who have episodes of severe depression or feel suicidal. This is the last resort for physicians. It appeared in 1930, it was overused and improvements to this technique have been done since 1980. Modern ECT is done with anesthesia. The patient receives an injection with a neuromuscular blocking agent before the treatment to prevent violent muscle contractions and abnormal heart rhythms. Researchers think that the electric shock triggers alterations in brain chemistry that directs to enhancing the patient's mood. ECT side effect is that causes temporary memory loss and confusion. ECT quickly interrupts an episode of mania or depression, but it does not have a long-term effect like lithium and other mood stabilizers. It is not very popular since "it is certainly more complicated to go through general anesthesia two or three times a week from two to four weeks than taking medication" (Mondimore 2006). Another treatment is the transcranial magnetic stimulation (TMS) which applies rapid pulses of a magnetic field to the head. It appears to have an antidepressant effect. It is not effective with everyone and it is a treatment that needs more research. No electricity passes through the skull like in ECT, a tiny electrical current is induced through the brain tissue and no anesthesia is applied. It seems to be a very promising treatment, since it activates the pre-frontal lobe to interrupt depression. It is also mentioned that it needs to be explored and research more. It seems the tiny current stimulates the area of the brain where the coil is placed. TMS has been used for mapping the brain. The patient is awake during the treatment. Another cutting edge approach is the Vagal Nerve Stimulation (VNS) approved by the FDA. It has been used to treat epilepsy since 1997. It is like a pacemaker device that is implanted in the vagus nerve that begins in the base of the brain and continues down the neck, into the chest and abdomen. This device transports regularly tiny electrical signals to stimulate the vagus nerve. It has been effective in 
reducing and eliminating depression in half the patients that have used it. The speed and organization of thoughts appears to get better after the treatment. Among the side effects are feeling a tickle during the electrical stimulation, induced panic and hypomania. But, it has the potential to switch BD into mania, and potentially made the patient unresponsive to medication if the mania episode gets worse. Not a recommended treatment by me. The Magnetic Resonance Spectroscopy Imaging (MRSI) that has been used to map the brain in patients, they have reported to feel happier after a scan. That happier mood lasted hours and days, but more serious research needs to be done. It can also trigger the mania episode in BD patients. It is not available and FDA has not licensed MRSI as a appropriate treatment for BPD. In extreme cases, people with BD benefit from hospitalization to obtain the psychiatric treatment needed to stay safe while their moods are stabilized.

\section{Teaching Strategies for Students with Bipolar Disorder}

A child with BP means the child has a significant health impairment that needs ongoing medical management. The medicines applied that treat it can have an impact in the student's attendance, focus and concentration. They may experience sensitivity to light, sound and anxiety. Tolerance and patience to ignore minor negative behavior seems to be the first and major attribute a teacher must display, since the positive behavior expected needs to be emphasized, encouraged, praised and recognized in order to help the student monitor own behavior. Few distractions, shorter assignments are highly recommended. Resource room assistance should be available at the last period of the day to enable to the student to record, review and catch up on anything he or she missed during the first periods of the day. Other accommodations for memory retention and help the child stay focused include to let the student have another set of school books left at home, giving the child with a hard copy of notes taken in class, also breaking long projects and assignments into smaller parts. Children with bipolar disorder need at least a person and place that they can have as resource when they begin to feel overwhelmed with the intensity of their emotions. The student should receive prior notice for routine changes and transitions. It is advisable to schedule the most challenging tasks when the student is at his/her best to do work. The most important element for the success of these students is the way adults respond to them. The best strategy for defiance and aggression is to not take it personally and to not get involved in power struggles. We have to remain a positive model. We need to keep observing this child to notice when the student has low energy levels or high ones and communicate these observations to the parents and therapists. Sometimes it is an indication that a cycle or episode may be beginning. Whatever makes the child seem irritable should be shared to parents. These observations should help the teacher to avoid scenarios that will make the student irritable. Experts say that situations involving physical contact, and competition prompt aggressive behavior, therefore we should limit the time of the classes that are potential triggers such as gym, music, recess. Accommodations due to medication include letting the student keep a water bottle if the student is taking lithium, also unlimited access to the bathroom since the student might be experiencing diarrhea as a second effect from a mood stabilizer.

\section{Areas for Future Research}

Treatment is an area that needs to be explored thoroughly since there is no medication, treatment or a combination of both approaches at this moment that help to cure, prevent or make BPD disappear in its totality or prevent it for longer periods of time. Medication and other alternate treatments have two main effects, to control a lifetime condition, and some other stop the episodes for a short period of time. One of the most promising treatments without medication that must be researched is the MRI. It has been discovered by accident in 2010. The patients who experienced the benefits of the brain imaging where in a study which purpose was to investigate the effectives of some targeted medication. The scientists think that it was one kind of the magnetic pulses they use that caused that reaction. 23 of the 30 patients felt happier. What makes this attractive as a treatment is that of those 23, the ones who were not taking medication experienced a higher improvement in their mood than the ones who were taking medicines to treat BD. However, the duration of the positive response needs to be researched, as it could be categorized as either for short-term or long term treatment. Another aspect of this treatment that needs to be studied is the options with or without medication available and effective to prevent and/or control a potential mania episode before it can be triggered. Lithium seems to have a tremendous positive impact for BD patients. Even though, blood tests need to be done to regulate the amount of that chemical in the body, and damage to the liver is considered the most serious side effect; lithium seems to help change the size of the brain, by increasing its volume. The chemical targets the $\mathrm{G}$ protein inside the neuron, brain neuroplasticity with lithium helps the brain make new connections and regenerate the old neurons making new combinations. In a study with mice that were induced with lithium and later killed, their brains showed "a $25 \%$ increase in the number of dividing cells in a structure of the hippocampus called the dentate gyrus-a clear indication of neurogenesis" (Block 2004). It seems that a defective neurogenesis in the brain is correlated to depression. The effect of lithium in the BD patient's brain helps increase the volume of the hippocampus while he/she experiences a happier mood. These are areas I think must be explored for patients having a variety of effective treatments with longterm results. However, the trend for future research I found in many articles is scientists trying to track a gene that is related to mood disorders. Everybody is trying to find some predictable pattern or path to treat it before the first episode happens. In a study which results were reported on January 17, 2010 in the journal Nature Genetics a group of international scientists studied data from five different genome-wide association studies (GWAS) which made 13,600 people, and to validate the results of the original group 
conducted 3 additional more samples with 4,677 people. The results of the study showed that variations of chromosome 3 are associated with BD. "Ultimately, findings such as these may lead to identification of common biological pathways that may play a role in both unipolar and bipolar illness and suggest strategies for better treatment." (Asher 2010). The hope is to finding a gene that can be traced to family history to detect BD at an early age and prevent the manifestation of this mood disorder during late adolescence years or early twenties.

\section{References}

1. Jules A (2010) Same Genes Suspected in Both Depression and Bipolar Illness.

2. Increased Risk May Stem From Variation in Gene On/Off Switch. National Institute of Mental Health.

3. Will B (2004) Can Lithium Benefit Brain Health? Life Enhancement Magazine.

4. Bob B (2010) Psychiatry and Human Behavior at Warren Alpert Medical School at Brown University. Bipolar Disorder (DSM-IVTR \#296.0-296.89).

5. Wess B (2006) The Bipolar Handbook. New York: Penguin Group.

6. Conrad M, Dryden-Edwards R, Marks J (2010) What is the role of the locus coeruleus in stress?. Medicinenet.com.
7. Daniel (2008) The Elusive Genetics of Bipolar Disorder.

8. Dolan (2010) DNA Learning Center \& The National Institute of Mental Health.

9. Neuropathology of Bipolar Disorder with Ellen Leibenluft. (video file 8) Retrieved from Dolan DNA Learning Center \& The National Institute of Mental Health. (2010, February 8).

10. Neuropathology of Bipolar Disorder with Ellen Leibenluft (video file 9).

11. James L (2011) Integrative Treatment of Bipolar Disorder: A Review of the Evidence and Recommendations. Psychiatric Times pp: 58-63.

12. Machado-Vieira R, Manji HK, Zarate CA Jr (2009) The role of lithium in the treatment of Bipolar Disorder: convergent evidence for neurotrophic effects as a unifying hypothesis. Bipolar Disord 2: 92-109.

13. Mondimore, Mark F (2006). Bipolar Disorder. Maryland: John Hopkins Press.

14. Packer,

Leslie E (2002) Accommodating Students with Mood Liability: De pression and Bipolar Disorder.

15. Stephanie S (2012) Through the Ages, It's been there. BP Magazine Volume 8: 2.

16. Wolf W (2010) Effects of Serotonin on Bipolar Disorder. LiveStrong.com 